\title{
Successful resuscitation of amniotic fluid embolism applying a new classification and management strategy
}

\author{
Shinya Yufune ${ }^{1 *}$, Motoshi Tanaka ${ }^{1}$, Ryosuke Akai ${ }^{1}$, Yasushi Satoh ${ }^{1}$, Kenichi Furuya ${ }^{2}$, Katsuo Terui ${ }^{3}$,
} Naohiro Kanayama ${ }^{4}$ and Tomiei Kazama ${ }^{1}$

\begin{abstract}
Amniotic fluid embolism (AFE) is a rare but life-threatening maternal emergency caused by the entry of amniotic fluid contents into the maternal circulation. The clinical manifestations of AFE are heterogeneous, leading to misdiagnosis or treatment delay. Kanayama and colleagues distinguished the cardiopulmonary collapse type (or classic type) from the disseminated intravascular coagulation (DIC) type of AFE on the basis of the presence of uterine atony and DIC in the latter prior to cardiopulmonary failure. We report a case of DIC-type AFE successfully treated by blood volume replacement and coagulation therapy. The patient was scheduled for elective cesarean delivery because of a previous cesarean section and moyamoya disease. Delivery was uneventful, but massive vaginal bleeding without clotting and ensuing hypovolemic shock occurred $4 \mathrm{~h}$ later. She was transferred to the operating room for emergency laparotomy, but sustained a cardiac arrest. The patient was successfully resuscitated and a hysterectomy performed. During surgery, the patient received fresh frozen plasma, platelets, fibrinogen, and antithrombin concentrate. In cardiopulmonary collapse type AFE, cardiopulmonary resuscitation without delay is important. In the present case of DIC-type AFE, however, early supplementation of clotting factors and platelets was critical for patient survival.
\end{abstract}

Keywords: Amniotic fluid embolism; Disseminated intravascular coagulation (DIC); Uterine atony; Cesarean delivery

\section{Background}

Amniotic fluid embolism (AFE), a severe maternal reaction to amniotic fluid contents entering the circulation, occurs in only $2-8$ of every 100,000 deliveries $[1,2]$. However, AFE has a high mortality of $21.6 \%$ in the United States and $24.3 \%$ in Japan [3, 4]. The risk factors associated with AFE include advanced maternal age, placental abnormalities, operative deliveries, eclampsia, polyhydramnios, cervical lacerations, and uterine rupture [5]. The three classic AFE symptoms are acute hypoxia, severe hypotension or cardiac arrest, and coagulopathy, which generally occur suddenly during labor (or pregnancy termination) or shortly after delivery [6]. However, AFE has a wide spectrum of manifestations, and patients do not always follow the classic clinical course. On the

\footnotetext{
* Correspondence: yufune@r4.dion.ne.jp

'Department of Anesthesiology, National Defense Medical College, 3-2

Namiki, Tokorozawa, Saitama 359-8513, Japan

Full list of author information is available at the end of the article
}

basis of this clinical heterogeneity, it was recently suggested that AFE may involve disseminated intravascular coagulation (DIC), uterine atony, and (or) cardiopulmonary collapse [4]. We present a case of AFE with cardiac arrest arising from DIC following elective cesarean delivery.

\section{Case presentation}

A 38-year-old woman $(67.3 \mathrm{~kg}, 160 \mathrm{~cm}$ tall, and G3 P2) with moyamoya disease was scheduled for a repeat cesarean delivery. She gave us her history of frequent transient ischemic attacks during hyperventilation associated with moyamoya disease; therefore, a cesarean delivery under general anesthesia was planned to avoid ischemic attack during labor. She was not taking any herbal preparations or anticoagulants. Laboratory findings on admission were as follows: normal electrocardiogram and chest X-ray, hemoglobin $10.7 \mathrm{~g} / \mathrm{dL}$, platelet count $32.2 \times 10^{4} / \mu \mathrm{L}$, fibrin degradation product (FDP)

\section{实}


$6 \mu \mathrm{g} / \mathrm{mL}$, D-dimer $2.2 \mu \mathrm{g} / \mathrm{mL}$, fibrinogen $431 \mathrm{mg} / \mathrm{dL}$, no urinary glucose or protein. At 38 weeks and 1 day of gestation, cesarean delivery was performed under general anesthesia without complications. The patient's trachea was extubated and returned to the obstetrics ward.

Four hours after cesarean delivery, vaginal hemorrhage increased rapidly. The patient's systolic arterial pressure decreased to $80 \mathrm{mmHg}$ and heart rate (HR) increased to 148 beats/min. Ultrasound imaging and computed tomography (CT) revealed a large retrovesical hematoma. The patient was transferred to the operating room for emergency laparotomy, and general anesthesia was induced. A few minutes after skin incision, airway pressure increased suddenly, $\mathrm{SpO}_{2}$ declined gradually, arterial blood pressure became undetectable, and HR was absent. Immediate cardiopulmonary resuscitation was initiated. Spontaneous circulation resumed after administration of $1 \mathrm{mg}$ epinephrine, but additional bolus injections and continuous epinephrine infusion were required to maintain spontaneous circulation. The uterus was atonic and swollen to the size of a person's head. Although a B-Lynch suture was attempted, atonic hemorrhage remained uncontrolled and the decision to perform hysterectomy was made. A large amount of bloody fluid was obtained from the surgical site, and the blood did not clot. Bleeding from the nose, the oral cavity, and the tracheal tube was observed. Coagulation tests, including those for FDP, D-dimer, and fibrinogen, showed that fibrinogen had decreased to $<50 \mathrm{mg} / \mathrm{dL}$. We administered 44 units of packed red blood cells (PRBCs), 44 units of fresh frozen plasma (FFP), 55 units of platelets, $8 \mathrm{~g}$ of fibrinogen, and 3,000 international units (IU) of antithrombin concentrate during the surgery (In Japan, estimated volume per unit of blood products is as follows: PRBCs $140 \mathrm{~mL} / \mathrm{unit}$, FFP $120 \mathrm{~mL} / \mathrm{unit}$, platelet $20 \mathrm{~mL} / \mathrm{unit})$. Intraoperative anesthetic course and the result of coagulation tests are shown in Fig. 1. After hemodynamic conditions stabilized, hemorrhage from the nose, the oral cavity, and the tracheal tube gradually decreased, and the patient was transferred to the intensive care unit (ICU) without extubation.

Computed tomography after surgery revealed pulmonary embolism (PE) but no cerebral hemorrhage. Heparin therapy was initiated to maintain activated partial thromboplastin time (APTT) within the therapeutic target range of $50-70 \mathrm{~s}$. Results of intraoperative maternal serum analysis were as follows: zinc coproporphyrin-1 (Zn-CP1) of $4 \mathrm{pmol} / \mathrm{mL}$ (normal, $<1.6 \mathrm{pmol} / \mathrm{mL}$ ), sialylTn antigen (STN) $20.0 \mathrm{IU} / \mathrm{mL}$ (normal, $<46 \mathrm{IU} / \mathrm{mL}$ ), compliment factor 3 (C3) $58 \mathrm{mg} / \mathrm{dL}$ (normal, $80-140 \mathrm{mg} /$ $\mathrm{dL}$ ), C4 $9 \mathrm{mg} / \mathrm{dL}$ (normal, 11-34 mg/dL), and interleukin-8 (IL-8) $405 \mathrm{pg} / \mathrm{mL}$ (normal, <20 pg/mL). Increased Zn-CP1 and IL-8 with decreased C3 and C4 strongly suggested AFE [7].

The pathological findings from the uterus of this patient are shown in Fig. 2. The amniotic fluid-derived ingredients are not detected in the hematoxylin-eosin (HE) staining, but Anti-Pan Cytokeratin (AE1/AE3) stains detected amniotic fluid-derived fetal keratin (Fig. 2a) and alcian blue

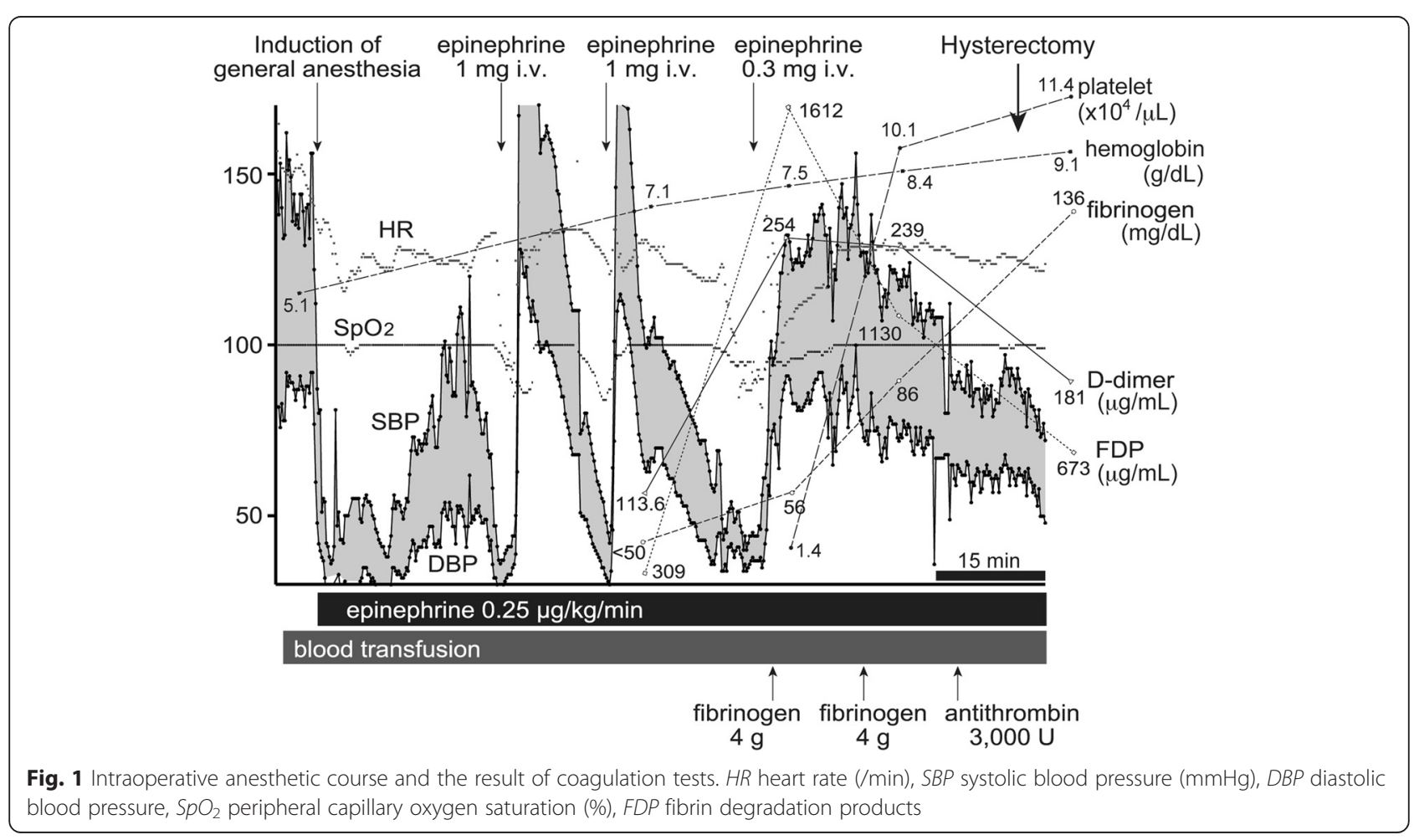



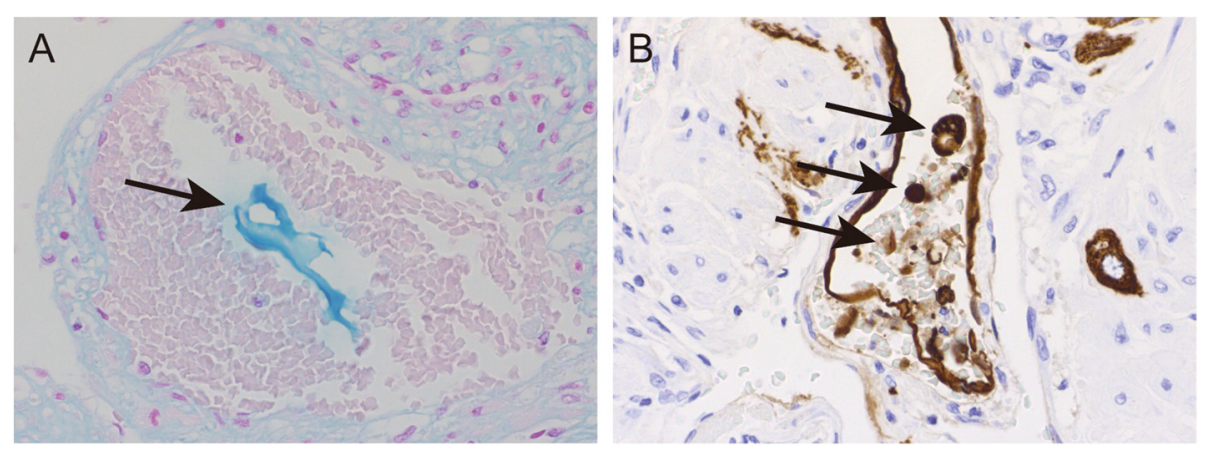

Fig. 2 Histological sections of the uterus. a Acid mucopolysaccharidic matter was observed in uterine vessels (black arrow), Alcian blue staining, 20x. b Immunohistochemically marked epithelial squames in uterine vessels (black arrows), Cytokeratin AE1/AE3 immunohistochemical staining, 20X

staining detected amniotic fluid-derived mucin (Fig. 2b) and edematous changes are observed in the uterus.

Seven days after the surgery, the patient's trachea was extubated and discharged from the ICU. Anticoagulant therapy was switched from heparin infusion to warfarin. Eighteen days after surgery, she was discharged from the hospital.

\section{Discussion}

Steiner and Lushbaugh (1941) first reported the cases of eight women who died unexpectedly from "obstetric shock" associated with fetal material in pulmonary vessels [8]. They concluded that pulmonary embolism from particulate matter in amniotic fluid was the most likely cause of death. Since then, many investigators have tried to clarify the pathophysiology of AFE, but the etiology remains controversial. Recent studies suggest that, in addition to mechanical obstruction of pulmonary vessels, AFE can also result from a systemic inflammatory response syndrome (SIRS), manifesting as anaphylaxis or septic shock [2]. Thus, Clark suggests the term "anaphylactoid syndrome of pregnancy" to better describe the pathophysiology of AFE [6].

The clinical manifestations of AFE include sudden hypoxia, severe hypotension (including cardiac arrest), and DIC. In the classic AFE type, acute dyspnea and desaturation appear during labor or shortly after delivery, followed by cardiopulmonary collapse and coagulopathy. However, we have observed many cases of forme fruste AFE in which one or more components of this symptom triad were minimal or absent. Kanayama et al. suggested that AFE can be separated into cardiopulmonary collapse and DIC types on the basis of clinical findings (Table 1) [4, 7]. In their report, the autopsies of five cardiopulmonary collapse-type patients revealed no remarkable changes in the uteri, whereas examination of four DIC-type patients showed amniotic components in all uteri, suggesting that the reaction of amniotic components to the uterus can cause uterine atony and DIC [7]. In typical DIC-type AFE, the patient shows uterine atony followed by DIC prior to cardiopulmonary symptoms; therefore, volume resuscitation and the supplementation of platelets and clotting factors are essential to save such patients.

To manage hemorrhage during surgery, transfusion of PRBCs is recommended to maintain oxygen transport at first, and coagulation tests and platelet count should be obtained before administering FFP and platelets [9]. However, many AFE patients require immediate FFP and platelet transfusion as well as packed red cells due to the

Table 1 New classification of amniotic fluid embolism

\begin{tabular}{|c|c|c|c|c|}
\hline & Initial symptoms & $\begin{array}{l}\text { Time from symptom } \\
\text { onset to cardiac arrest }\end{array}$ & Histology & Initial management \\
\hline \multirow{3}{*}{$\begin{array}{l}\text { Cardiopulmonary collapse } \\
\text { type (Classic type) }\end{array}$} & - Sudden dyspnea & \multirow{3}{*}{$\begin{array}{l}\text { Very short } \\
\text { (0-60 min in typical cases) }\end{array}$} & \multirow{3}{*}{$\begin{array}{l}\text { - Amniotic components } \\
\text { in pulmonary vessels }\end{array}$} & \multirow{3}{*}{$\begin{array}{l}\text { Cardiopulmonary resuscitation } \\
\text { including inotropes }\end{array}$} \\
\hline & $\begin{array}{l}\text { - Severe hypotension } \\
\text { (including cardiac arrest) }\end{array}$ & & & \\
\hline & - Seizure & & & \\
\hline \multirow[t]{3}{*}{ DIC type } & $\begin{array}{l}\text { - Massive bleeding } \\
\text { without clotting }\end{array}$ & \multirow[t]{3}{*}{ Several hours } & $\begin{array}{l}\text { - Amniotic components in } \\
\text { uterus and/or uterine vessels }\end{array}$ & \multirow{3}{*}{$\begin{array}{l}\text { Volume resuscitation including } \\
\text { supplement of platelets and } \\
\text { clotting factors }\end{array}$} \\
\hline & \multirow[t]{2}{*}{ - Uterine atony } & & - Thrombus in uterine vessels & \\
\hline & & & - Intestinal edema in uterus & \\
\hline
\end{tabular}


consumption of coagulation factors at the early stage of AFE. The blood transfusion strategy for AFE patients is thus similar to the massive transfusion protocol for trauma patients, in which disruption of hemostasis occurs because of dilution coagulopathy, inflammatory mediator activation, hyperfibriolysis, thrombocytopenia, and metabolic abnormalities [10]. In our case, we recognized that vaginal bleeding without clotting was an early sign of DIC-type AFE and promptly initiated transfusion of PRBCs and FFP (1:1), platelets, fibrinogen, and antithrombin concentrate.

The diagnosis of AFE is based primarily on clinical observation. In the past, the detection of fetal squamous cells in the maternal pulmonary circulation was considered a definitive diagnostic finding of AFE. However, a recent study detected fetal squamous cells in only $50 \%$ AFE patients during the aspiration of pulmonary artery blood [6], whereas another report found that the presence of squamous cells in the maternal circulation was not always associated with AFE [11]. Although there is no gold standard diagnostic marker for AFE, we believe that changes in a number of serum factors are strongly indicative of AFE, including Zn-CP1, STN, C3, C4, and IL-8. Meconium is rich in Zn-CP1 [12], and STN is a sugar chain of the mucin-type glycoprotein that recognizes mucin in the meconium/amniotic fluid [13]. We thus propose that STN and Zn-CP1 in the maternal circulation are suggestive of AFE [7]. Benson et al. reported that $\mathrm{C} 3$ and $\mathrm{C} 4$ levels in AFE patients were significantly lower than normal [14], suggesting that complement activation has an important role in the pathophysiology of AFE. Amniotic fluid components can also activate immune cells associated with allergic reactions, which produce large amounts of bradykinin and inflammatory cytokines such as IL-8 [7]. Recently, C1 estarase inhibitor activity (C1INH) was suggested as a prognostic marker of AFE [15]. In addition to serum factors, histological observation revealed edematous changes exist in the uterine smooth muscle and amniotic fluid components and fetal epithelial cells in the uterine vessels in this patient are suggestive of DIC-type AFE [7].

In this particular case patient, the reasons for cardiac arrest are unclear. The possibilities include superimposed cardiopulmonary type AFE and pulmonary thromboembolism. Although the patient showed no symptoms of deep vein thrombosis (DVT) before cesarean delivery, preoperative D-dimer levels were elevated; hence, DVT is possible. Interestingly, both serum FDP and D-dimer decreased promptly after administration of fibrinogen and antithrombin concentrate. The onset of cardiac arrest was more than $4 \mathrm{~h}$ after delivery, not sudden, and initial symptoms was massive bleeding without clotting, we considered cardiac arrest was due to hypovolemic shock. We cannot entirely exclude the classic type of AFE as the causation of cardiac arrest, although initial manifestation, change of serum factors, and histological observations in uterus are suggestive of DIC-type AFE. Appropriate treatment for DIC at the right time might lead to her recovery from DIC.

\section{Conclusions}

In conclusion, we describe a case of DIC-type AFE. Unlike cardiopulmonary type AFE, uterine atony and DIC were the initial symptoms. Early diagnosis and prompt administration of blood products, including clotting factors and platelets, are essential for patient recovery.

\section{Consent}

Written informed consent was obtained from the patient for publication of this case report and any accompanying images. A copy of the written consent is available for review by the Editor-in-chief of this journal.

\section{Abbreviations \\ AFE: amniotic fluid embolism; APTT: activated partial thromboplastin time: C1 INH: C1 estarase inhibitor activity; C3: compliment factor 3; \\ C4: compliment factor 4; CT: computed tomography; DBP: diastolic blood pressure; DIC: disseminated intravascular coagulation; DVT: deep vein thrombosis; FDP: fibrin degradation products; FFP: fresh frozen plasma; HE: hematoxylin-eosin; HR: heart rate; ICU: intensive care unit; \\ IL-8: interleukin-8; IU: international units; PE: pulmonary embolism; PRBCs: packed red blood cells; SBP: systolic blood pressure; SIRS: systemic inflammatory response syndrome; $\mathrm{SpO}_{2}$ : peripheral cappilary oxygen saturation; STN: sialyl-Tn antigen; Zn-CP1: zinc coproporphyrin-1.}

\section{Competing interests}

The authors declare that they have no competing interests.

\section{Authors' contributions}

SY, MT, and RA drafted the article. SY and YS acquired the data. KF, KT, and NK participated in design and coordination and helped to draft the manuscript. TK supervised and edited the manuscript. All authors discussed the results and commented on the manuscript. All authors read and approved the final manuscript.

\section{Acknowledgements}

We thank Hideyuki Shimazaki, MD, PhD (Associate professor of Laboratory Medicine, National Defense Medical College Hospital, Saitama, Japan) and Kuniaki Nakanishi, MD, PhD (Professor of Laboratory Medicine, National Defense Medical College Hospital, Saitama, Japan) for their excellent technical help with this case report. This work is attributed to and supported by the Department of Anesthesiology, National Defense Medical College. This case report has not been published elsewhere.

\section{Author details}

'Department of Anesthesiology, National Defense Medical College, 3-2 Namiki, Tokorozawa, Saitama 359-8513, Japan. ${ }^{2}$ Department of Obstetrics and Gynecology, National Defense Medical College, 3-2 Namiki, Tokorozawa, Saitama 359-8513, Japan. ${ }^{3}$ Department of Obstetric Anesthesiology, Saitama Medical Center, Saitama Medical University, 1981 Kamoda, Kawagoe, Saitama 350-8550, Japan. ${ }^{4}$ Department of Obstetrics and Gynecology, Hamamatsu University School of Medicine, 1-20-1, Handayama, Higashi-ku, Hamamatsu, Shizuoka 431-3192, Japan.

Received: 11 May 2015 Accepted: 22 June 2015

Published online: 27 August 2015 


\section{References}

1. Rath WH, Hoferr S, Sinicina I. Amniotic fluid embolism: an interdisciplinary challenge: epidemiology, diagnosis and treatment. Dtsch Arztebl Int. 2014;111:126-32

2. Clark SL. Amniotic fluid embolism. Obstet Gynecol. 2014;123:337-48.

3. Abenhaim HA, Azoulay L, Kramer MS, Leduc L. Incidence and risk factors of amniotic fluid embolisms: a population-based study on 3 million births in the United States. Am J Obstet Gynecol. 2008;199:49. e41-48.

4. Kanayama N, Inori J, Ishibashi-Ueda H, Takeuchi M, Nakayama M, Kimura S, et al. Maternal death analysis from the Japanese autopsy registry for recent 16 years: significance of amniotic fluid embolism. J Obstet Gynaecol Res. 2011;37:58-63.

5. Conde-Agudelo A, Romero R. Amniotic fluid embolism: an evidence-based review. Am J Obstet Gynecol. 2009;201:445. e441-413.

6. Clark SL, Hankins GD, Dudley DA, Dildy GA, Porter TF. Amniotic fluid embolism: analysis of the national registry. Am J Obstet Gynecol. 1995:172:1158-67. discussion 1167-1159.

7. Kanayama N, Tamura N. Amniotic fluid embolism: pathophysiology and new strategies for management. J Obstet Gynaecol Res. 2014;40:1507-17.

8. Steiner PE, Lushbaugh CC. Landmark article, Oct. 1941: maternal pulmonary embolism by amniotic fluid as a cause of obstetric shock and unexpected deaths in obstetrics. By Paul E. Steiner and C. C. Lushbaugh. JAMA. 1986;255:2187-203.

9. American Society of Anesthesiologists Task Force on Perioperative Blood T, Adjuvant T. Practice guidelines for perioperative blood transfusion and adjuvant therapies: an updated report by the American Society of Anesthesiologists Task Force on Perioperative Blood Transfusion and Adjuvant Therapies. Anesthesiology. 2006:105:198-208.

10. Pham HP, Shaz BH. Update on massive transfusion. Br J Anaesth 2013;111 Suppl 1:i71-82.

11. Lee W, Ginsburg KA, Cotton DB, Kaufman RH. Squamous and trophoblastic cells in the maternal pulmonary circulation identified by invasive hemodynamic monitoring during the peripartum period. Am J Obstet Gynecol. 1986;155:999-1001.

12. Kanayama N, Yamazaki T, Naruse H, Sumimoto K, Horiuchi K, Terao T. Determining zinc coproporphyrin in maternal plasma-a new method for diagnosing amniotic fluid embolism. Clin Chem. 1992:38:526-9.

13. Kobayashi $\mathrm{H}$, Ohi $\mathrm{H}$, Terao T. A simple, noninvasive, sensitive method for diagnosis of amniotic fluid embolism by monoclonal antibody $\mathrm{TKH}-2$ that recognizes NeuAc alpha 2-6GalNAc. Am J Obstet Gynecol. 1993;168:848-53.

14. Benson MD, Kobayashi H, Silver RK, Oi H, Greenberger PA, Terao T. Immunologic studies in presumed amniotic fluid embolism. Obstet Gynecol. 2001;97:510-4.

15. Tamura N, Kimura S, Farhana M, Uchida T, Suzuki K, Sugihara K, et al. C1 esterase inhibitor activity in amniotic fluid embolism*. Crit Care Med. 2014;42:1392-6.

\section{Submit your manuscript to a SpringerOpen ${ }^{\circ}$ journal and benefit from:}

- Convenient online submission

- Rigorous peer review

- Immediate publication on acceptance

Open access: articles freely available online

- High visibility within the field

- Retaining the copyright to your article

Submit your next manuscript at $>$ springeropen.com 